\title{
Overdiagnosis: what it is and what it isn't
}

\section{John Brodersen, ${ }^{1,2}$ Lisa M Schwartz, ${ }^{3}$ Carl Heneghan, ${ }^{4}$ Jack William O'Sullivan, ${ }^{4}$ Jeffrey K Aronson, ${ }^{4}$ Steven Woloshin ${ }^{3}$}

${ }^{1}$ Centre of Research \&

Education in General Practice, Department of Public Health, University of Copenhagen, Faculty of Health Sciences, Copenhagen, Denmark

${ }^{2}$ Region Zealand, Primary Health Care Research Unit ${ }^{3}$ Center for Medicine and the Media, The Dartmouth Institute for Health Policy and Clinical Practice, Dartmouth Medical School, Lebanon, New Hampshire, USA ${ }^{4}$ Centre for EvidenceBased Medicine, Nuffield Department of Primary Care Health Sciences, University of Oxford, Oxford, UK

Correspondence to: Professor John Brodersen, Centre of Research \& Education in General Practice, Department of Public Health, University of Copenhagen, Faculty of Health Sciences, Copenhagen 1014, Denmark; jobr@sund.ku.dk

\section{CrossMark}

To cite: Brodersen J, Schwartz LM, Heneghan C, et al. BMJ Evidence-Based Medicine 2018;23:1-3.
Why then, can one desire too much of a good thing?

William Shakespeare, As You Like It (1600)

Rosalind's question, as she is about to marry Orlando, is purely rhetorical-she thinks that one cannot desire too much of a good thing. Nevertheless, trite though it may be, it is true that one can sometimes have it. It is certainly true of healthcare and has been referred to as 'too much medicine', although because of potential confusion with 'too much medication' a better term might be 'too much healthcare'. This includes too much screening of asymptomatic individuals, too much investigation of those with symptoms, too much reliance on biomarkers, too many quasi-diseases, too much diagnosis, often leading to too much treatment, sometimes cost-ineffective, medicines that are too costly and too rapidly approved for marketing, too many adverse reactions, and too much inappropriate monitoring. And too much healthcare implies too little effective healthcare.

An older term, 'overdiagnosis' has been used to refer to a more restricted set of items. And although the term can be traced back as far as $1955,{ }^{2}$ it is still difficult to define satisfactorily.

Broadly, overdiagnosis means making people patients unnecessarily, by identifying problems that were never going to cause harm or by medicalising ordinary life experiences through expanded definitions of diseases.

Overdiagnosis has two major causes: overdetection and overdefinition of disease. While the forms of overdiagnosis differ, the consequences are the same: diagnoses that ultimately cause more harm than benefit. Confusion about what constitutes overdiagnosis undermines progress to a solution. Here we aim to draw boundaries around what overdiagnosis is and to exclude what it is not.

\section{What it is}

Overdetection refers to the identification of abnormalities that were never going to cause harm, abnormalities that do not progress, that progress too slowly to cause symptoms or harm during a person's remaining lifetime, or that resolve spontaneously. ${ }^{3}$ Increasing use of high-resolution diagnostic technologies increases the risk of such overdetection. For example, high-resolution CT angiography can identify small subsegmental pulmonary emboli that may not need treatment. ${ }^{4}$

The emergence of sophisticated self-testing technologies, greater access to tests, and in some cases commercial incentives, has further propagated overdetection. The more tests you order, the more likely you are to diagnose a 'disease. ${ }^{56}$ This is particularly problematic when there is little evidence that early detection improves patient outcomes. Consider the example of thyroid cancer in South Korea. From 1999 to 2008, the incidence of thyroid cancer increased 6.4 -fold, ${ }^{7}$ but 95\% of these cancers were small $(<20 \mathrm{~mm})$, and they were detected mainly through screening. ${ }^{7}$ The mortality from thyroid cancer remained essentially unchanged over the same period. ${ }^{7}$ Use of advanced imaging also leads to overdetection by finding incidentalomas-'surprise' abnormalities unrelated to the original reason for doing the test, for example, when a chest CT done to follow-up on a pulmonary nodule detects a small adrenal adenomas. Overdetection-from whatever causeis a problem, because it is not possible to know which abnormalities are likely to progress. Epidemiological evidence showing a large rise in, for example, early forms of cancer or smaller abdominal aortic aneurysm, without similar reductions in advanced disease or death can be signals of overdiagnosis through overdetection. ${ }^{8} 9$

Overdefinition occurs by two mechanisms: lowering the threshold for a risk factor without evidence that doing so helps people feel better or live longer and by expanding disease definitions to include patients with ambiguous or very mild symptoms. An example of lowering the threshold would be changing high blood pressure from a systolic blood pressure of $>150$ to $>130$ for all adults. Treating risk factors as diseases and lowering the thresholds for risk factor-based diagnoses has dramatically increased the prevalence of many diseases such as the 'epidemic of prediabetes. ${ }^{10}$ By definition, these 'new' patients are at lower risk than those diagnosed under earlier definitions. The harmful consequences of overdiagnosis through overdefinition come from labelling and treatments (including lifestyle changes) that offer little, if any, benefit (given the low levels of risk) but that can have important physical, psychological, social, and financial consequences.

Overselling is an insidious tactic for promoting overdefinition. What characterises overselling is that the supposed 'diseases' are unpleasant experiences most people have from time to time. For example, most people have experienced trouble sleeping, sadness, or difficulty focusing. Overselling means moving the line separating normal from abnormal, so that people with milder and 
milder symptoms get diagnosed. For a minority of people, these symptoms are intense or debilitating. But for most, the symptoms are mild or fleeting. While the former may benefit from diagnosis and medical treatment (in this example, for insomnia, depression, or attention deficit hyperactivity disorder (ADHD)), the latter may not. Disease mongering is a synonymous term, historically reserved for broadening of disease that is especially questionable and typically done to sell more drugs (it should be noted that diagnosis may help people with more severe symptoms). Disease mongering has been a central strategy in prominent marketing campaigns for conditions such as low testosterone of ageing ('low t'), restless legs syndrome, binge eating disorder, adult ADHD, chronic dry eye disease, and too short eyelashes.

\section{What it isn't}

Overdiagnosis is not a false-positive result. False positives are abnormalities that turn out not to be diseases after further investigation. In overdiagnosis, the abnormality meets the currently agreed criteria for pathological disease (eg, microscopic criteria for cancer), but the disease detected is not destined to cause symptoms or death. There are rare reports of women in whom abnormal screening mammography suggested pathology, who were treated with lobectomy or mastectomy; however, the removed tissue showed no malignancy (ie, the test was a false positive). Those women were not subject to overdiagnosis, but they were certainly overtreated.

Overdiagnosis is not synonymous with overtreatment, whether unnecessary or overaggressive. Overdiagnosis typically leads to overtreatment, but not always. Furthermore, overtreatment can occur without overdiagnosis. Overtreatment occurs when the best scientific evidence demonstrates that a treatment provides no benefit for the diagnosed condition. For example, middle ear infections in children and bronchitis in adults are often correctly diagnosed but overtreated with ineffective antibiotics. Distinguishing overtreatment caused by lack (or ignorance) of the evidence from overtreatment caused by overdiagnosis is important, since the drivers and solutions may differ in important ways.

Overdiagnosis is also not synonymous with overtesting. Overtesting (sometimes referred to as overuse or overutilisation) can, but does not always, increase the risk of overdiagnosis, but the risk increases proportionately with the degree of overuse. For example, there is a relationship between how many prostate-specific antigen (PSA) tests are ordered in general practice and the incidence of prostate cancer, ${ }^{11}$ and between the number of male patients on a general practitioner's (GP) list with a diagnosis of cancer and the number of PSA tests ordered. ${ }^{12}$ GPs who undertake many PSA tests have many more male patients with overdiagnoses of prostate cancer, because the mortality rate is the same across clinics irrespective of the number of tests ordered. $^{12}$

Overdiagnosis is not misdiagnosis, but sometimes this distinction is muddy. A clear-cut misdiagnosis is a completely wrong diagnosis. For example, a boy who cannot focus in class and exhibits disruptive behaviour may be thought to have ADHD. However, he turns out to have dyslexia, not ADHD. Muddier cases typically occur in cancer, where an overdiagnosed cancer could be considered a misdiagnosis of progressive cancer that requires treatment. Because neoplastic pathology at the time of diagnosis is just a snapshot, it cannot perfectly distinguish the clinically relevant from the overdiagnosed cancers (eg, one Gleason grade prostate cancer might be destined to progress while another of the same grade is not). If new biomarkers, genes or antibodies could perfectly distinguish neoplastic pathology, a diagnosis of 'cancer' not destined to progress could be considered to be a misdiagnosis, rather than an overdiagnosis.

\section{Conclusion}

Overdiagnosis is one of the most harmful and costly problems in modern healthcare. It often triggers a cascade of overtreatment, although the two are not synonymous. To prevent and minimise overdiagnosis, we need more studies on the natural history of diseases, watchful waiting trials of very early/small or ambiguous abnormalities, studies of the effects of diagnostic language, intervention studies on known drivers of overdiagnosis, and studies of how to involve patients in decisions about diagnostic strategies. And we need to ensure that new disease definitions are based on evidence, ${ }^{13}$ not financial interests. ${ }^{14}$

Describing what overdiagnosis is and what it isn't is essential, so that more attention and resources are directed towards preventing it.

Contributors JB drafted the first version of the manuscript. All authors contributed to revisions with important intellectual content and approved the final version of the manuscript. JB is the guarantor.

Competing interests JB is a member of the board and scientific committee of the Overdiagnosis Conference with international partners as the BMJ plus Dartmouth, Bond and Oxford universities based on a non-profit model. JB is also the host of the next Overdiagnosis Conference in Copenhagen August 2018. LMS and SW have served as medical experts in testosterone litigation and were the cofounders of Informulary, Inc., a company that provided data about the benefits and harms of prescription drugs, which ceased operations in December 2016. The Dartmouth Institute jointly runs the Overdiagnosis Conference. $\mathrm{CH}$ has received expenses and fees for his media work including BBC Inside Health. He holds grant funding from the NIHR, the NIHR School of Primary Care Research, The Wellcome Trust and the WHO. CEBM jointly runs the Evidence Live Conference with the BMJ and the Overdiagnosis Conference. JWO is funded by the Clarendon Fund, University of Oxford. He has also received income from Oxford University Hospitals for clinical work and hold grants from the National Institute for Health Research and the Primary Care Research Trust. He is a member of the Junior Scientific Board of the Overdiagnosis Conference. JKA is an associate editor of the EBM journal, a member of the CEBM (see $\mathrm{CH}$ above), president emeritus of the British Pharmacological Society, and an emeritus fellow of Green-Templeton College, Oxford; he has written articles and edited textbooks on adverse drug reactions and has been an expert witness in cases of drug toxicity.

Provenance and peer review Commissioned; internally peer reviewed.

- Article author(s) (or their employer(s) unless otherwise stated in the text of the article) 2018. All rights reserved. No commercial use is permitted unless otherwise expressly granted.

\section{References}

1. Moynihan R, Smith R. Too much medicine? BMJ 2002;324:859-60.

2. Case RA, Lea AJ. Mustard gas poisoning, chronic bronchitis, and lung cancer; an investigation into the possibility that poisoning by mustard gas in the 1914-18 war might be a factor in the production of neoplasia. $\mathrm{Br} \mathrm{J}$ Prev Soc Med 1955;9:62-72. 
3. Brodersen J, Schwartz LM, Woloshin S. Overdiagnosis: how cancer screening can turn indolent pathology into illness. APMIS 2014;122:683-9.

4. Wiener RS, Schwartz LM, Woloshin S. When a test is too good: how CT pulmonary angiograms find pulmonary emboli that do not need to be found. BMJ 2013;347:f3368.

5. Ahn HS, Kim HJ, Welch HG. Korea's thyroid-cancer "epidemic" - screening and overdiagnosis. $N$ Engl J Med 2014;371:1765-7.

6. McCoy RG, Van Houten HK, Ross JS, et al. HbA1c overtesting and overtreatment among US adults with controlled type 2 diabetes, 2001-13: observational population based study. BMJ 2015;351:h6138.

7. Park S, Oh CM, Cho H, et al. Association between screening and the thyroid cancer "epidemic" in South Korea: evidence from a nationwide study. BMJ 2016;355:i5745.

8. Esserman L, Shieh Y, Thompson I. Rethinking screening for breast cancer and prostate cancer. JAMA 2009;302:1685-92.
9. Johansson M, Hansson A, Brodersen J. Estimating overdiagnosis in screening for abdominal aortic aneurysm: could a change in smoking habits and lowered aortic diameter tip the balance of screening towards harm? BMJ 2015;350:h825.

10. Yudkin JS, Montori VM. The epidemic of pre-diabetes: the medicine and the politics. BMJ 2014;349:g4485.

11. Mukai TO, Bro F, Pedersen KV, et al. Use of prostate-specific antigen testing. Ugeskr Laeger 2010;172:696-700.

12. Hjertholm P, Fenger-Grøn M, Vestergaard M, et al. Variation in general practice prostate-specific antigen testing and prostate cancer outcomes: an ecological study. Int J Cancer 2015;136.

13. Doust J, Vandvik PO, Qaseem A, et al. Guidance for modifying the definition of diseases: a checklist. JAMA Intern Med 2017;177:1020-5.

14. Moynihan RN, Cooke GP, Doust JA, et al. Expanding disease definitions in guidelines and expert panel ties to industry: a cross-sectional study of common conditions in the United States. PLoS Med 2013;10:e1001500. 